OPEN ACCESS

Edited by:

Yong Teng,

Emory University, United States

Reviewed by:

Eliska Svastova,

Slovak Academy of Sciences, Slovakia

Martin Francis Lavin,

The University of Queensland,

Australia

${ }^{*}$ Correspondence:

Jiafu Feng

jiafufeng@aliyun.com

Lin Yu

xiaoyulin918@163.com

${ }^{+}$These authors have contributed equally to this work

Specialty section: This article was submitted to Cancer Metabolism,

a section of the journal

Frontiers in Oncology

Received: 25 June 2021 Accepted: 23 September 2021 Published: 15 October 2021

Citation:

Xu B, Chen Y, Chen X, Gan L, Zhang Y, Feng $J$ and $Y u L$ (2021) Metabolomics Profiling Discriminates Prostate Cancer From Benign Prostatic Hyperplasia Within the ProstateSpecific Antigen Gray Zone.

Front. Oncol. 11:730638. doi: 10.3389/fonc.2021.730638

\section{Metabolomics Profiling Discriminates Prostate Cancer From Benign Prostatic Hyperplasia Within the Prostate-Specific Antigen Gray Zone}

\author{
Bei Xu ${ }^{1 \dagger}$, Yan Chen ${ }^{2 \dagger}, X_{i}$ Chen $^{3}$, Lingling Gan ${ }^{1}$, Yamei Zhang ${ }^{1}$, Jiafu Feng ${ }^{1 *}$ and Lin Yu ${ }^{1 *}$ \\ ${ }^{1}$ Department of Clinical Laboratory, Mianyang Central Hospital, School of Medicine, University of Electronic Science and \\ Technology of China, Mianyang, China, ${ }^{2}$ Department of Clinical Pharmacy, Sichuan Cancer Hospital \& Institute, Sichuan \\ Cancer Center, School of Medicine, University of Electronic Science and Technology of China, Chengdu, China, \\ ${ }^{3}$ Department of Application Support Center, SCIEX Analytical Instrument Trading Co., Shanghai, China
}

Objective: Prostate cancer ( $\mathrm{PCa}$ ) is the second most common male malignancy globally. Prostate-specific antigen (PSA) is an important biomarker for PCa diagnosis. However, it is not accurate in the diagnostic gray zone of $4-10 \mathrm{ng} / \mathrm{ml}$ of PSA. In the current study, the performance of serum metabolomics profiling in discriminating PCa patients from benign prostatic hyperplasia $(\mathrm{BPH})$ individuals with a PSA concentration in the range of $4-10 \mathrm{ng} / \mathrm{ml}$ was explored.

Methods: A total of 220 individuals, including patients diagnosed with $\mathrm{PCa}$ and $\mathrm{BPH}$ within PSA levels in the range of $4-10 \mathrm{ng} / \mathrm{ml}$ and healthy controls, were enrolled in the study. Liquid chromatography coupled with tandem mass spectrometry (LC-MS/MS)based non-targeted metabolomics method was utilized to characterize serum metabolic profiles of participants. Principal component analysis (PCA) and partial least squares discriminant analysis (PLS-DA) methods were used for multivariate analysis. Receiver operating characteristic (ROC) curve analysis was performed to explore the diagnostic value of candidate metabolites in differentiating PCa from BPH. Correlation analysis was conducted to explore the relationship between serum metabolites and common clinically used fasting lipid profiles.

Results: Several differential metabolites were identified. The top enriched pathways in PCa subjects such as glycerophospholipid and glycerolipid metabolisms were associated with lipid metabolism. Lipids and lipid-like compounds were the predominant metabolites within the top 50 differential metabolites selected using fold-change threshold $>1.5$ or $<2$ / 3 , variable importance in projection $(\mathrm{VIP})>1$, and Student's t-test threshold $p<0.05$. Eighteen lipid or lipid-related metabolites were selected including 4-oxoretinol, anandamide, palmitic acid, glycerol 1-hexadecanoate, DL-dihydrosphingosine, 2methoxy-6Z-hexadecenoic acid, 3-oxo-nonadecanoic acid, 2-hydroxy-nonadecanoic acid, N-palmitoyl glycine, 2-palmitoylglycerol, hexadecenal, D-erythro-sphingosine C-15, $N$-methyl arachidonoyl amine, 9-octadecenal, hexadecyl acetyl glycerol, 1-(9Zpentadecenoyl)-2-eicosanoyl-glycero-3-phosphate, 3Z,6Z,9Z-octadecatriene, and 
glycidyl stearate. Selected metabolites effectively discriminated PCa from BPH when PSA levels were in the range of $4-10 \mathrm{ng} / \mathrm{ml}$ (area under the curve $(A \cup C)>0.80$ ). Notably, the 18 identified metabolites were negatively corrected with total cholesterol (TC), low-density lipoprotein cholesterol (LDL-C), and Apo-B levels in PCa patients; and some were negatively correlated with high-density lipoprotein cholesterol (HDL-C) and Apo-A levels. However, the metabolites were not correlated with triglycerides (TG).

Conclusion: The findings of the present study indicate that metabolic reprogramming, mainly lipid metabolism, is a key signature of PCa. The 18 lipid or lipid-associated metabolites identified in this study are potential diagnostic markers for differential diagnosis of PCa patients and BPH individuals within a PSA level in the gray zone of 4$10 \mathrm{ng} / \mathrm{ml}$.

Keywords: prostate cancer, prostate-specific antigen, untargeted metabolomics, lipid metabolism, candidate biomarkers

\section{INTRODUCTION}

Globally, prostate cancer ( $\mathrm{PCa}$ ) is the second most prevalent male malignancy, accounting for more than 1 million new cases and more than 0.35 million deaths (1). Currently, diagnosis and localization of PCa are mainly based on digital rectal examination (DRE) and assessment of serum prostate-specific antigen (PSA) levels and final verification through transrectal ultrasound-guided prostate biopsy (TRUSPB) (2). PSA is an androgen-regulated serine protease produced and secreted by prostate epithelial cells, with approximate range of normal levels at $0-1.5 \mathrm{ng} / \mathrm{ml}$ (3). Elevated levels of PSA $(>4 \mathrm{ng} / \mathrm{ml})$ are associated with PCa (1). However, high PSA levels are also observed in individuals with benign prostatic hyperplasia $(\mathrm{BPH})$, prostatitis, and prostate injury $(4,5)$. More evidence demonstrates that the positive predictive value of PSA is averagely $21 \%$ within the gray zone of $4-10 \mathrm{ng} / \mathrm{ml}$ (2), implying that the PSA approach has a poor specificity for PCa diagnosis. Invasive TRUSPB-based histological examination is currently the main diagnostic approach for $\mathrm{PCa}$; however, it is not routinely recommended for patients due to the tedious procedure and associated significant discomfort and complications. Moreover, most individuals without $\mathrm{PCa}$ undergo unnecessary TRUSPB owing to the poor specificity of the PSA test ultimately developing complications $(6,7)$. Therefore, it is imperative to develop novel effective and minimally invasive detection biomarkers for accurate PCa screening, mainly at the gray zone $4-10 \mathrm{ng} / \mathrm{ml}$.

Aberrant cancer metabolism is a newly recognized hallmark of various malignancies $(8,9)$. Studies have previously used metabolomics, which is the systematic study of the metabolites, to elucidate chemical fingerprints that specific cellular states and processes leave behind, thus providing in-depth understanding of health and disease. Metabolomics analysis of biological fluids is an effective and minimally invasive technology for disease monitoring, and a potential diagnostic non-invasive tool $(8,10)$. Several cancers including colorectal cancer (11), pancreatic cancer (12), and gastric cancer (13) exhibit significantly altered metabolite concentrations between cancer patients and healthy controls (HCs). Additionally, previous studies have explored the potential role of metabolites in urine, blood (serum or plasma), seminal fluid, or (tumor) tissues in PCa progression $(1,14,15)$. Evidences show that healthy prostate cells favor citrate synthesis over citrate utilization and mainly rely on glucose oxidation to provide energy, thus resulting in citrate accumulation (15-17). Accumulation of zinc in benign prostate cells inhibits activity of $\mathrm{m}$-aconitase (ACO), which catalyzes conversion of citrate to isocitrate in the tricarboxylic acid (TCA) cycle $(16,18)$. However, prostate cells have no capacity to accumulate zinc following neoplastic transformation. As a result, $\mathrm{m}$-aconitase activity and citrate oxidation are restored, which are accompanied by subsequent decrease in citrate accumulation and increase in ATP production $(18,19)$. Increased lipid biosynthesis, which is important for cellular proliferation and intercellular signaling, is a classical metabolic reprogramming in malignant transformation. To achieve increased lipid metabolism, citrate is converted to acetyl-CoA (a precursor for lipogenesis and cholesterogenesis) in the cytosol (18). Recent findings show upregulation of expression of several key enzymes associated with cholesterol and fatty acid synthesis, which are regulated by androgen; and the enzymes exhibit increased activity in PCa cells (18). Studies on plasma metabolites in circulation in PCa are mainly focused on glycine, alanine, sarcosine, and a few lipid metabolites $(1,14,15)$. However, these biomolecules cannot discriminate patients with $\mathrm{PCa}$ and $\mathrm{BPH}$ in the PSA gray zone of $4-10 \mathrm{ng} / \mathrm{ml}$. Moreover, the results on metabolites are not consistent across different studies. The differences are attributed to application of different methods for metabolomics analysis, differences in experimental designs, and varying characteristics of the study participants (20). Metabolomics is a rapidly growing new field with significant methodological and technical hurdles; therefore, more definitive studies focusing on metabolic pathways potentially altered during prostate tumorigenesis and progression should be conducted.

The currents study sought to explore novel potential serum metabolic biomarkers in PCa patients within the gray zone of 4- 
$10 \mathrm{ng} / \mathrm{ml}$ through liquid chromatography coupled with tandem mass spectrometry (LC-MS/MS) analysis. Previous studies mainly focused on alterations of metabolites in PCa patients in high PSA levels (always $>10 \mathrm{ng} / \mathrm{ml}$ ) compared with $\mathrm{BPH}$ individuals. In the present study, levels of novel metabolic candidates in PCa patients were compared with the levels in $\mathrm{BPH}$ controls within the gray zone of $4-10 \mathrm{ng} / \mathrm{ml}$. The findings of the current study showed that the identified metabolites effectively distinguished two common disease conditions with PSA levels in the clinical diagnostic gray zone, therefore, have a high potential for clinical decision making (Figure 1).

\section{MATERIALS AND METHODS}

\section{Study Design and Participants}

A total of $74 \mathrm{PCa}$ patients, $74 \mathrm{BPH}$ patients, and 72 healthy subjects were enrolled in the current prospective pilot study. The study protocol was approved by the Medical Ethics Committee of Mianyang Central Hospital. Patients were diagnosed with PCa through TRUSPB method but had not undergone any treatment. Men diagnosed with BPH were used as the disease control group. Hematoxylin and eosin (H\&E) staining and immunohistochemical assay of alpha-methylacyl-CoA racemase (AMACR, P504S), highmolecular-weight cytokeratin (HMWCK), and P63 antigens were done on the prostate specimens. Briefly, specimens were formalinfixed and embedded in paraffin. Serial sections of $5-\mu \mathrm{m}$ thickness were cut and stained with H\&E. Sections were processed for immunohistochemistry, as follows. Sections were dewaxed in xylene and hydrated in graded ethanol and water. Antigen retrieval was obtained by boiling in $0.01 \mathrm{~mol} / \mathrm{L}$ of citrate buffer (pH 6.0) for $20 \mathrm{~min}$. Sections were then respectively incubated with the primary antibodies at a dilution of 1:100 for $30 \mathrm{~min}$ at room temperature. Immunostaining was performed with a streptavidinbiotin complex kit (Beyotime Biotechnology Co. Ltd, Shanghai, China). After incubation with diaminobenzidine (DAB) as a chromogen, the specimens were counterstained with Harris hematoxylin and cover slipped. Staining was observed using the light microscope at $\times 200$ magnification (Olympus, Tokyo, Japan). The represent pathological section staining is shown in

\section{Supplementary Figure S1.}

Participant characteristics are presented in Table 1. The range of serum PSA concentrations of patients with PCa or BPH was 4-10 $\mathrm{ng} / \mathrm{ml}$. Analysis showed no significant differences among the three groups except for PSA (referring to total PSA (TPSA) in this study) and Gleason scores. PCa and BPH groups showed significantly higher prostate PSA levels (both $p<0.001$ ) compared with the levels in the HC group. Notably, PSA levels in the PCa and BPH groups were not significantly different. The number of PCa patients with GS4-GS9 was $18,25,17,9,3$, and 2, respectively.

\section{Sample Collection and Analysis}

Venous blood was collected from all participants between 6:00 and 10:00 a.m. after undergoing overnight fasting to eliminate diet-induced variations. Serum was obtained from blood samples by centrifugation (3,000 rpm for $15 \mathrm{~min}$ ). All clinical laboratory tests were performed within $2 \mathrm{~h}$ after serum collection and were stored at $-80^{\circ} \mathrm{C}$ for subsequent analysis.

TABLE 1 | Characteristics of the individuals included in this study.

\begin{tabular}{lccc}
\hline & $\begin{array}{c}\text { HC group } \\
(\mathbf{n}=\mathbf{7 2})\end{array}$ & $\begin{array}{c}\text { BPH group } \\
(\mathbf{n}=\mathbf{7 4})\end{array}$ & $\begin{array}{c}\text { PCa group } \\
\mathbf{( n = 7 4 )}\end{array}$ \\
\hline Age (years) & $62.1 \pm 8.7$ & $62.2 \pm 12.2$ & $64.1 \pm 8.2$ \\
BMl (kg/m $\left.{ }^{2}\right)$ & $27.1 \pm 6.1$ & $26.1 \pm 5.7$ & $26.4 \pm 6.4$ \\
Prostate volume (ml) & $47.3 \pm 26.6$ & $52.6 \pm 18.9$ & $46.9 \pm 25.7$ \\
FPSA (ng/ml) & $0.66 \pm 0.51$ & $1.19 \pm 0.73$ & $1.06 \pm 0.41$ \\
TPSA (ng/ml) & $2.24 \pm 1.09$ & $7.68 \pm 2.81^{\# \# \#}$ & $8.69 \pm 2.16^{\star \star \star}$ \\
Tumor Gleason score & $\mathrm{NA}$ & $\mathrm{NA}$ & $5(4-9)$ \\
\hline
\end{tabular}

BMI, body mass index; FPSA, free prostate-specific antigen; TPSA, total prostate-specific antigen; $\mathrm{HC}$, healthy control; $\mathrm{BPH}$, benign prostatic hyperplasia; $\mathrm{PCa}$, prostate cancer. $B P H$ group compared with HC group, ${ }^{\# \# ~} \mathrm{p}<0.001$; PCa group compared with HC group, ${ }^{* * *} \mathrm{p}<0.001$.
Recruitment and informed consent of patients diagnosed with PCa and BPH showing PSA levels in grey zone of 4-10 $\mathrm{ng} / \mathrm{mL}$ and healthy controls

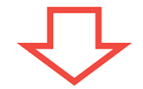

Metabolic profiles of serum samples analyzed by LC-MS/MS-based metabolomics approach

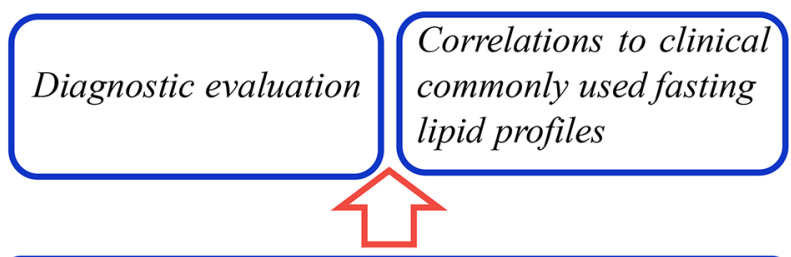

18 lipid-associated metabolites were retained

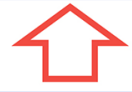

Potential metabolic biomarkers identification and metabolic pathway enrichment according to $F C>1.5$ or $<2 / 3, V I P>1$ and $P<0.05$

FIGURE 1 | The workflow of the analysis steps. 
Concentrations of triglycerides (TG), total cholesterol (TC), high-density lipoprotein cholesterol (HDL-C), and low-density lipoprotein cholesterol (LDL-C) were evaluated using the same serum sample through LC-MS/MS analysis.

\section{Sample Preparation for Liquid Chromatography-MS/MS Analysis}

A mixture of serum samples $(190 \mu \mathrm{l})$, internal standard $(10 \mu \mathrm{l})$ (a mixture of clenbuterol and chloramphenicol for the positive and negative ion modes, respectively), and methanol-acetonitrile $(1: 1 \mathrm{v} / \mathrm{v})(800 \mu \mathrm{l})$ was incubated at $-20^{\circ} \mathrm{C}$ for $1 \mathrm{~h}$. The mixture was then vortexed and centrifuged, and the supernatant was obtained as previously described (13). The resulting solutions were filtered through a $0.22-\mu \mathrm{m}$ microporous membrane. A $5 \mu \mathrm{l}$ aliquot of the filtrate was transferred into an autosampler and injected into the LC-MS/MS system for metabolomics analysis. Aliquots of all serum samples $(10 \mu \mathrm{l})$ were pooled to prepare quality control (QC) samples. Pretreatment of the QC samples was performed following the same procedure as that of the study samples. QC samples were introduced every 10 samples in the analytical sequence to evaluate the reliability of the large-scale metabolomics analysis (21).

\section{Instrumentation and Liquid Chromatography-MS/MS Conditions}

Analysis was performed using an Agilent ${ }^{\circledR} 1290$ Infinity II UPLC system (Agilent Technologies Inc., USA) coupled to a Triple TOF 5600+ mass spectrometer system (AB Sciex, Framingham, MA). A Waters ACQUITY HSS T3 C18 column $(100 \times 2.1 \mathrm{~mm}$, i.d. $1.8 \mu \mathrm{m}$ ) was used for separation of components. Continuous evaluation of the full-scan survey MS data was performed by an Acquisition software (Analyst TF1.7, AB Sciex) using preselected criteria for acquisition of MS/MS spectra. The mobile phase comprised water with $0.1 \%(\mathrm{v} / \mathrm{v})$ formic acid (solvent A) and acetonitrile with $0.1 \%(\mathrm{v} / \mathrm{v})$ formic acid (solvent B). The gradient for the mobile phase was as follows: a linear gradient of 99\% A over initial-1.2 min; 99\%-30\% A over 1.2-4.5 min; 30\%-1\% A over 4.5-13.0 min; 1\% A over 13.0-16.5 min; 1\%-99\% A over 16.5-16.6 min; and 99\% A over 16.6-20.0 min. The flow rate was set at $0.30 \mathrm{ml} / \mathrm{min}$. The column temperature was set as $30^{\circ} \mathrm{C}$. An electrospray ionization (ESI) source was used with positive and negative modes for the mass spectrometer system. Electrospray source parameters were set as follows: curtain gas (CUR), 35 psi; ion source gas 1 (GS1), 55 psi; ion source gas 2 (GS2), 55 psi; temperature (TEM), $550^{\circ} \mathrm{C}$; declustering potential (DP), $\pm 80 \mathrm{~V}$; collision energy (CE), \pm $10 \mathrm{~V}$; and accumulation time (AT), $0.15 \mathrm{~s}$.

\section{Identification of Differential Metabolites and Metabolic Pathway Analysis}

Analyst TF (version 1.7.1, AB Sciex, USA) qualitative analysis software was used for acquisition and processing of the raw data. A metabolomics data processing workflow was then established with a serial set of processes including peak picking, quality assurance, normalization, missing value imputation, transformation, and scaling. The processed molecular weights of the metabolites (molecular weight error $<20 \mathrm{ppm}$ ) were confirmed, matched, and annotated using a standard database, custom databases (Metlin, MassBank, LipidMaps, Mzclound, HMDB, and ONE-MAP databases), and other integrated databases to achieve accurate metabolite characterization.

Metabolites with fold-change threshold $>1.5$ or $<2 / 3$, variable importance in projection (VIP) $>1$, and Student's t-test threshold $p<0.05$ were selected as differential metabolites for group discrimination. Metabolic pathways associated with differential metabolites were explored using Kyoto Encyclopedia of Genes and Genomes (KEGG) and MetaboAnalyst databases through comparison between the change in metabolite ion intensity and the corresponding controls.

The metabolomics community proposed defined metrics for assessing the confidence of an annotation (22). All metabolites identified in the current study were defined "annotation" (levels 2) and did not require exhaustive analytical validation. This was according to formal definitions of metabolite annotation and identification developed by the Chemical Analysis Working Group of the Metabolomics Standards Initiative (MSI) (23).

\section{Statistical Analysis}

All statistical analyses were performed with SPSS 25.0 software (International Business Machines Corp., USA). Normally distributed continuous data were expressed as mean \pm standard deviation (SD), and comparisons between two groups were performed using Student's t-test. Non-normally distributed variables were expressed as the median and interquartile range (IQR) and compared using the Mann-Whitney $U$ tests. Differences among multiple groups were compared using oneway ANOVA if the variances were equal. Welch's approximate analysis of variance was applied followed by Dunnett's T3 test when the variances were uneven. Pearson's or Spearman's bivariate correlation analysis were performed for normal or skewed distribution to explore the relationship between selected metabolites parameters and commonly used fasting lipid profiles. A $p$-value less than 0.05 denoted statistical significance.

Multivariate analysis was performed using SIMCA 15.0.2 software (Umetrics AB, Umea, Sweden). LC-MS/MS data were subjected to principal component analysis (PCA) using an unsupervised non-targeted approach, to visualize metabolome variation among groups. Metabolome differences between sample pairs were maximized using a supervised classification method with unit variance scaling of partial least squares discriminant analysis (PLS-DA), and key variables contributing to the classification were identified according to their VIP (24). Permutation tests with 200 random variables were conducted to avoid data overfitting in the PLS-DA model. Diagnostic performance of differential metabolites was evaluated using receiver operating characteristic (ROC) curves. The area under the curve (AUC) was recorded as a measure of diagnostic accuracy and compared among group. The trade-offs between sensitivity and specificity for each variable were aggregated. AUC of 1.0 denoted $100 \%$ sensitivity and specificity, indicating perfect assignment, whereas an AUC of 0.5 indicated an unreliable test (gray line) (25). SPSS 25.0 (International Business Machines 
Corp., USA) was used for correlation analysis between metabolites and clinical parameters.

\section{RESULTS}

\section{Multivariate Statistical Analysis of Metabolites}

LC-MS/MS is an important technique that generates twodimensional profiles of constituent compounds over retention time (RT) and uses mass-to-charge ratio $(\mathrm{m} / \mathrm{z})$ for metabolomics analysis of biological samples. LC-MS/MS method was used for analysis of 220 serum samples and 20 QC samples in the positive (ESI+) and negative (ESI-) ion modes. Processed data comprising RT, exact mass, and peak intensity were subjected to multivariate statistical analysis.

General clusters, trends, or outliers among the observations were visualized using PCA. Score plots demonstrated a direct image of observational distributions. A distinct classification was achieved for the $\mathrm{PCa}, \mathrm{BPH}$, and $\mathrm{HC}$ groups in positive ion mode (Figures 2A, C). The results showed effective separation of the principal components of $\mathrm{PCa}, \mathrm{BPH}$, and $\mathrm{HC}$ groups in positive ion mode. However, $\mathrm{PCa}, \mathrm{BPH}$, and $\mathrm{HC}$ groups were not effectively clustered under the negative ion mode (Figures 2B, D); therefore, multivariate statistical analysis was needed to further explore their relationship. The pooled QC samples clustered together in the PCA plots in both positive and negative ion modes (Figures $2 \mathrm{C}, \mathrm{D}$ and Supplementary Figure S2), indicating stability and repeatability of the analysis system.

Moreover, PLS-DA was performed to further explore the different metabolic profiles, as the method has a better discriminative power compared with that of PCA and to identify potential biomarkers. Distinct clustering was observed for the $\mathrm{PCa}$ and $\mathrm{BPH}$ groups in both positive (Figure $\mathbf{3 A}$ ) and negative (Figure $3 \mathbf{C}$ ) ion modes, indicating accurate separation of the two groups. The $\mathrm{R}^{2} \mathrm{X}, \mathrm{R}^{2} \mathrm{Y}$, and $\mathrm{Q}^{2}$ (cum) parameters in PLS-DA method were mainly applied for model evaluation. The parameters of modeling, $\mathrm{R}^{2} \mathrm{X}, \mathrm{R}^{2} \mathrm{Y}$, and $\mathrm{Q}^{2}$ (cum) in the positive ion mode were established as $0.23,0.80$, and 0.72 , respectively. The $R^{2} X, R^{2} Y$, and $Q^{2}$ (cum) for the negative mode were $0.36,0.82$, and 0.75 . High values of $\mathrm{Q}^{2}$ indicated high accuracy of the PLS-DA model. A 200 times' random
A

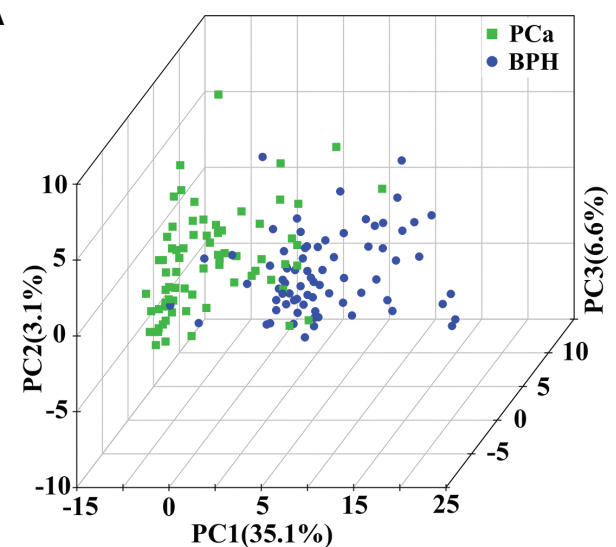

C

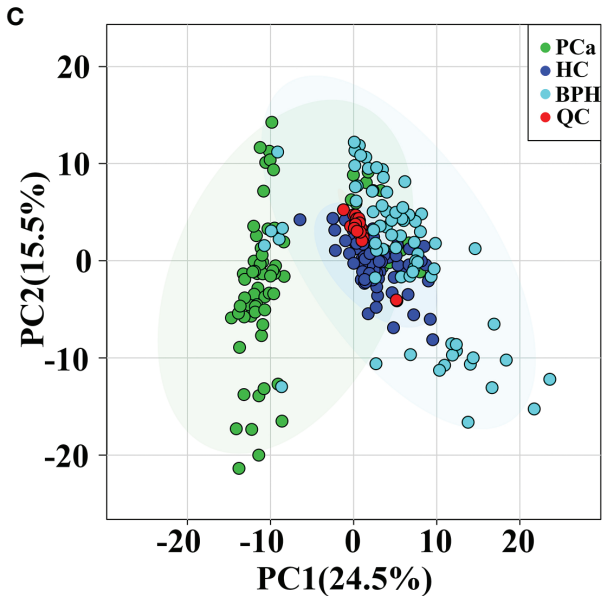

B

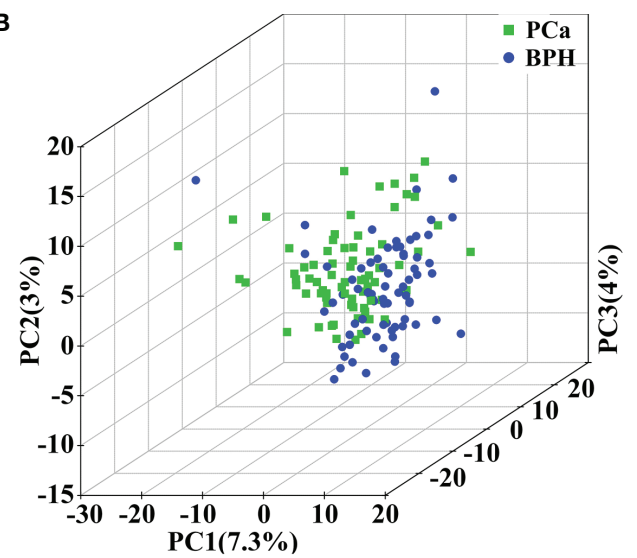

D

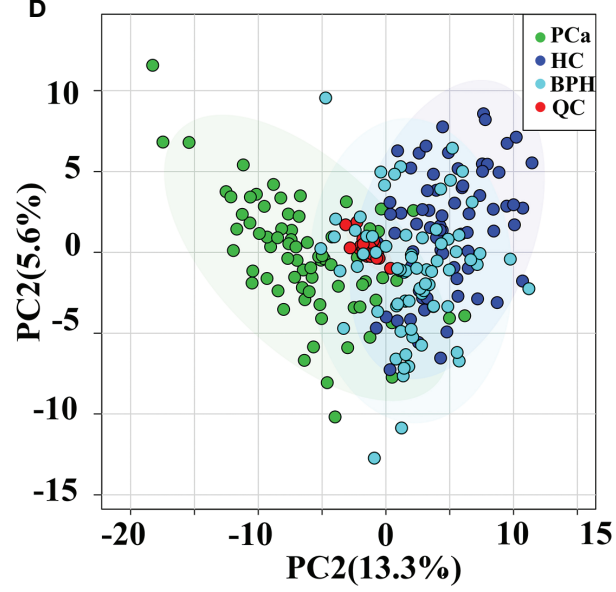

FIGURE 2 | PCA score plots in positive (A) and negative (B) ion modes between PCa and BPH groups. PCA score plots of QC samples in positive (C) and negative (D) ion modes among PCa, BPH, and HC groups. PCA, principal component analysis; PCa, prostate cancer; BPH, benign prostatic hyperplasia; QC, quality control; $\mathrm{HC}$, healthy control. 

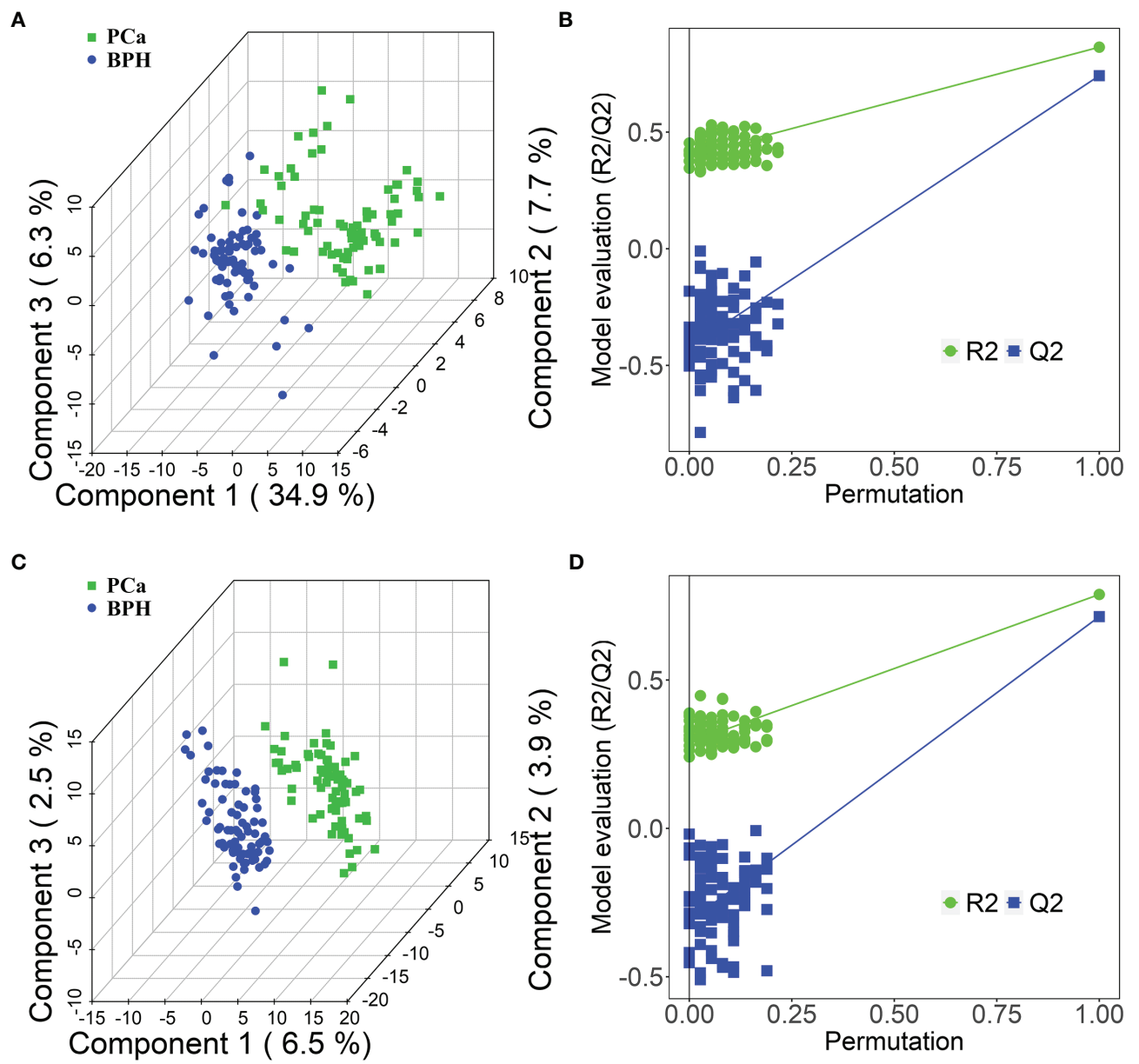

FIGURE 3 | PLS-DA score plots in positive (A) and negative (C) ion modes between PCa and BPH groups. PLS-DA permutation test plots in positive (B) and negative (D) ion modes between PCa and BPH groups. The criterion for evaluating whether there is overfitting in the PLS-DA model is that the regression line at a blue Q2 point crosses or is less than 0 from the abscissa. PLS-DA, partial least squares discriminant analysis; PCa, prostate cancer; BPH, benign prostatic hyperplasia.

permutation test was performed to explore the overfitting of the supervised PLS-DA models. The Y-intercepts of $\mathrm{Q}^{2}$ distributions were less than zero in both positive and negative ion modes (Figures 3B, D), indicating reliability of the established PLS-DA model. Moreover, distinct clustering of the $\mathrm{HC}$ and $\mathrm{PCa}$ or $\mathrm{BPH}$ groups in both positive and negative ion modes was observed (Supplementary Figures S3A-H). In summary, PCA and PLSDA models indicated significant distinction among $\mathrm{PCa}, \mathrm{BPH}$, and $\mathrm{HC}$ groups and were highly effective in characterizing serum metabolites.

\section{Identification of Potential Metabolites and Pathways}

Peaks were aligned, and missing values were eliminated from the MS/MS data, resulting in 6,891 and 7,868 peaks in the ESI+ and ESI- modes, respectively. Qualitative identification was conducted using publicly available databases, standard compounds databases, and several integrated databases. A total of 1,755 metabolites were identified in the positive ion mode, and
963 metabolites were identified in the negative ion mode. Subsequently, 362 different metabolites were selected using a fold-change threshold $>1.5$ or $<2 / 3$, VIP $>1$, and Student's t-test threshold $p<0.05$. Heat maps of the 20 differential metabolites detected in the positive and negative modes showed distinct clustering for each group, which was consistent with the orthogonal PLS-DA (OPLS-DA) results (Figures 4A, B). The most abundant class of metabolites was lipids and lipid-like molecules, including anandamide, DL-dihydrosphingosine, 4oxoretinol, and palmitic acid.

In addition, the differential metabolites were enriched in metabolic pathways. Pathway impact values were acquired as cumulative percentage of the matched metabolite nodes. $p$ Values were calculated by enrichment analysis based on false discovery rate (FDR) $[-\log (p)$ value]. Results of metabolic pathway analysis showed that the differential metabolites in PCa were significantly mainly enriched in glycerophospholipid metabolism, glycerolipid metabolism, arachidonic acid metabolism, nicotinate and nicotinamide metabolism, and 
A

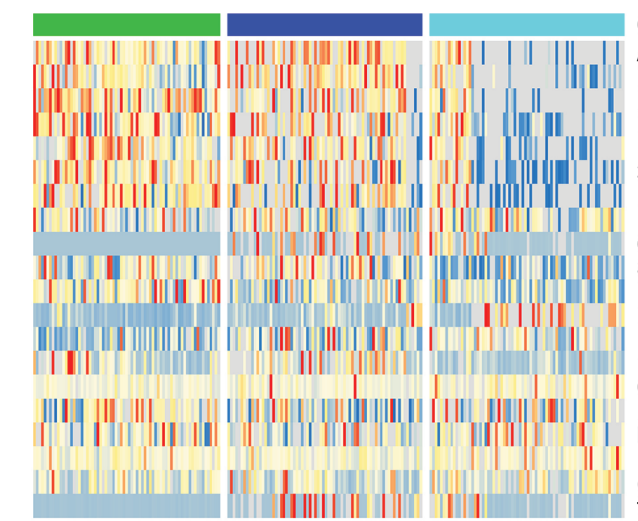

B

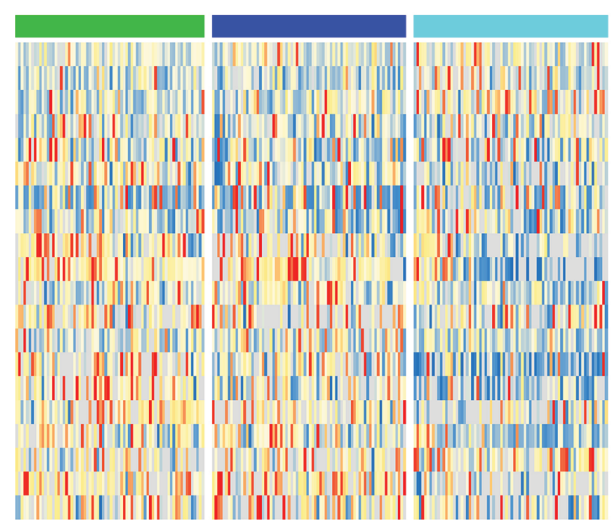

Class

Anandamide

DI-Dihydrosphingosine

D-erythro-Sphingosine C-15

$\mathrm{N}$-methyl arachidonoyl amine

PA(15:1(9Z)/20:0)

3Z,6Z,9Z-Octadecatriene

Phenylethylamide 359

PC 38:6

Oleamide

SM 34:2

PC(14:0/18:2(9Z,12Z))

$\mathrm{N}$-Glycosyl-L-asparagine

PC 34:1

1-(5-Phosphoribosyl)imidazole-4-acetate

Chol-11-Enic Acid

PC 39:1e

Lyso PC(18:4(6Z,9Z,12Z,15Z))

PGF2 $\alpha$; diethyl amide

(2R)-2-Hydroxy-2-methylbutanenitrile

trans-9-Octadecenoic acid

Class

Chenodeoxycholic acid sulfate

7-Ketodeoxycholic acid

Latanoprost (free acid)-d4

Acetaminophen glucuronide

Acetamino $28: 7$

17-Hydroxypregnenolone sulfate

2-Aminophenol sulphate

2-Acetoxy-6-pentadecylbenzoic acid

4-Oxoretinol

Palmitic acid

(9R,10R)-Dihydroxyoctadecanoic acid

Octadecanedioic acid

3-Indoleacrylic acid

19-hydroxy-17-oxoandrost-5-en-3- $\beta$-yl sulfate

19-hydroxy-1

LPG 9:0

Dihydrophaseic acid

PC 34:2

2,2'-Dithenyl sulfide

Sphingosine-1-phosphate

FIGURE 4 | Differential metabolite heat maps in positive (A) and negative (B) modes. The columns represent samples, the rows represent metabolites, and the relative content of the metabolites is displayed by color. The heat map shows differential metabolites among $\mathrm{HC}$, $\mathrm{BPH}$, and $\mathrm{PCa}$ groups. $\mathrm{HC}$, healthy control; $\mathrm{BPH}$, benign prostatic hyperplasia; PCa, prostate cancer.

biotin metabolism pathways compared with those in the $\mathrm{BPH}$ group. Notably, glycerophospholipid metabolism was the top significantly enriched pathway in PCa at the gray zone of 4-10 $\mathrm{ng} / \mathrm{ml}$. Moreover, lipid metabolism-related pathways were the most significantly enriched metabolic pathways. Detailed information on the pathways is presented in Table $\mathbf{2}$ and Supplementary Table S1. Significantly enriched metabolic pathways and the related metabolites between PCa and $\mathrm{HC}$ groups are presented in Supplementary Table S2.

TABLE 2 | Significantly altered metabolic pathways between PCa and BPH groups with PSA levels at gray zone of $4-10 \mathrm{ng} / \mathrm{ml}$.

\begin{tabular}{lcccc}
\hline Pathway name & KEGG.id & - $\log (\boldsymbol{p})$ & Impact & Hits \\
\hline Glycerophospholipid metabolism & hsa00564 & 5.18 & 0.42 & 6 \\
Glycerolipid metabolism & hsa00561 & 3.32 & 0.22 & 3 \\
Arachidonic acid metabolism & hsa00590 & 0.67 & 0.31 & 2 \\
Nicotinate and nicotinamide metabolism & hsa00760 & 0.66 & 0.19 & 1 \\
Biotin metabolism & hsa00780 & 0.96 & 0.15 & 1 \\
\hline
\end{tabular}

Impact, impact value of metabolic pathway determined by topology analysis; Hits, the number of differential metabolites matching the pathway; $P C a$, prostate cancer; $B P H$, benign prostatic hyperplasia; PSA, prostate-specific antigen; KEGG, Kyoto Encyclopedia of Genes and Genomes.

\section{Receiver Operating Characteristic Analysis of Selected Metabolites Discriminating Prostate Cancer Patients From Benign Prostatic Hyperplasia Patients}

A total of 18 lipid or lipid-like metabolites (4-oxoretinol, anandamide, palmitic acid, glycerol 1-hexadecanoate, DLdihydrosphingosine, 2-methoxy-6Z-hexadecenoic acid, 3-oxononadecanoic acid, 2-hydroxy-nonadecanoic acid, $N$-palmitoyl glycine, 2-palmitoylglycerol, hexadecenal, D-erythro-sphingosine C-15, N-methyl arachidonoyl amine, 9-octadecenal, hexadecyl acetyl glycerol, 1-(9Z-pentadecenoyl)-2-eicosanoyl-glycero-3phosphate [PA (15:1(9Z)/20:0)], 3Z,6Z,9Z-octadecatriene, and glycidyl stearate) were selected as candidate biomarkers for early diagnosis of $\mathrm{PCa}$ at the gray zone of $4-10 \mathrm{ng} / \mathrm{ml}$ using the following criteria: variable VIP $>2$, fold change (FC) $>1.5$ or $<2 / 3$, and $p<0.05$ (Table 3). The normalized intensity peak areas of the selected metabolites are presented in Figure 5. The levels of the 18 metabolites were significantly lower in PCa patients compared with the corresponding levels in $\mathrm{BPH}$ (all $p<0.001$ ).

ROC analysis of each biomarker was performed to their predictive value in PCa (Table 3 and Figure 6). The results 
TABLE 3 | Detection of 18 selected lipid metabolites as potential biomarkers for the diagnosis of PCa with PSA levels at gray zone of 4-10 ng/ml.

\begin{tabular}{|c|c|c|c|c|c|c|c|}
\hline Biomarker & FC & VIP & $p$-Value & AUC $(95 \% \mathrm{Cl})$ & Se $(\%)$ & Sp (\%) & Trend (cancer) \\
\hline 4-Oxoretinol & 0.65 & 2.71 & 6.51E-12 & $0.811(0.738-0.870)$ & 71.62 & 77.03 & $\downarrow^{\star \star \star}$ \\
\hline Anandamide & 0.31 & 2.65 & 2.27E-22 & $0.890(0.828-0.935)$ & 85.14 & 90.54 & $\downarrow^{\star \star \star}$ \\
\hline Palmitic acid & 0.28 & 2.59 & $5.88 \mathrm{E}-21$ & $0.878(0.814-0.926)$ & 79.73 & 91.89 & $\downarrow^{\star \star \star}$ \\
\hline Glycerol 1-hexadecanoate & 0.22 & 2.56 & $3.43 \mathrm{E}-20$ & $0.873(0.808-0.922)$ & 77.03 & 93.24 & $\downarrow^{\star \star \star}$ \\
\hline DL-Dihydrosphingosine & 0.59 & 2.22 & $5.25 \mathrm{E}-14$ & 0.838 (0.769-0.893) & 74.32 & 93.24 & $\downarrow^{\star \star \star}$ \\
\hline 2-Methoxy-6Z-hexadecenoic acid & 0.25 & 2.67 & 1.02E-22 & $0.889(0.827-0.935)$ & 82.43 & 91.89 & $\downarrow^{\star \star \star}$ \\
\hline 3-Oxo-nonadecanoic acid & 0.20 & 2.63 & $9.70 \mathrm{E}-22$ & $0.881(0.818-0.928)$ & 81.08 & 90.54 & $\downarrow^{\star \star \star}$ \\
\hline 2-Hydroxy-nonadecanoic acid & 0.24 & 2.61 & $2.98 \mathrm{E}-21$ & $0.884(0.821-0.931)$ & 81.08 & 91.89 & $\downarrow^{\star \star \star}$ \\
\hline N-Palmitoyl glycine & 0.23 & 2.58 & $1.60 \mathrm{E}-20$ & $0.862(0.795-0.913)$ & 81.08 & 90.54 & $\downarrow^{\star \star \star}$ \\
\hline 2-Palmitoylglycerol & 0.22 & 2.55 & $1.06 \mathrm{E}-19$ & $0.848(0.780-0.902)$ & 79.73 & 90.54 & $\downarrow^{\star \star \star}$ \\
\hline Hexadecenal & 0.32 & 2.51 & 4.03E-19 & $0.841(0.772-0.896)$ & 79.73 & 90.54 & $\downarrow^{\star \star \star}$ \\
\hline D-Erythro-sphingosine C-15 & 0.33 & 2.37 & $5.44 \mathrm{E}-17$ & $0.871(0.807-0.921)$ & 77.03 & 90.54 & $\downarrow^{\star \star \star}$ \\
\hline N-Methyl arachidonoyl amine & 0.42 & 2.30 & $1.68 \mathrm{E}-15$ & $0.853(0.785-0.906)$ & 85.14 & 75.68 & $\downarrow^{\star \star \star}$ \\
\hline 9-Octadecenal & 0.23 & 2.27 & $6.16 \mathrm{E}-15$ & $0.853(0.786-0.906)$ & 74.32 & 90.54 & $\downarrow^{\star \star \star}$ \\
\hline Hexadecyl acetyl glycerol & 0.25 & 2.24 & $5.78 \mathrm{E}-14$ & $0.833(0.763-0.889)$ & 78.38 & 89.19 & $\downarrow^{\star \star \star}$ \\
\hline PA(15:1(9Z)/20:0) & 0.44 & 2.14 & $4.89 \mathrm{E}-13$ & 0.839 (0.769-0.894) & 75.68 & 89.19 & $\downarrow^{\star \star \star}$ \\
\hline 3Z,6Z,9Z-Octadecatriene & 0.27 & 2.07 & $2.24 \mathrm{E}-11$ & $0.800(0.726-0.861)$ & 81.08 & 77.03 & $\downarrow^{\star \star \star}$ \\
\hline Glycidyl stearate & 0.22 & 2.05 & $1.88 \mathrm{E}-11$ & $0.804(0.731-0.865)$ & 79.73 & 79.73 & $\downarrow^{\star \star \star}$ \\
\hline
\end{tabular}

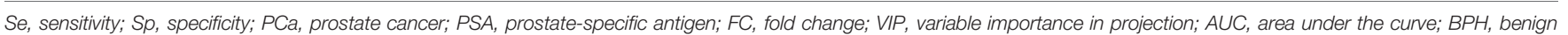
prostatic hyperplasia.

PCa group compared with BPH group, ${ }^{* *} \mathrm{p}<0.001$.

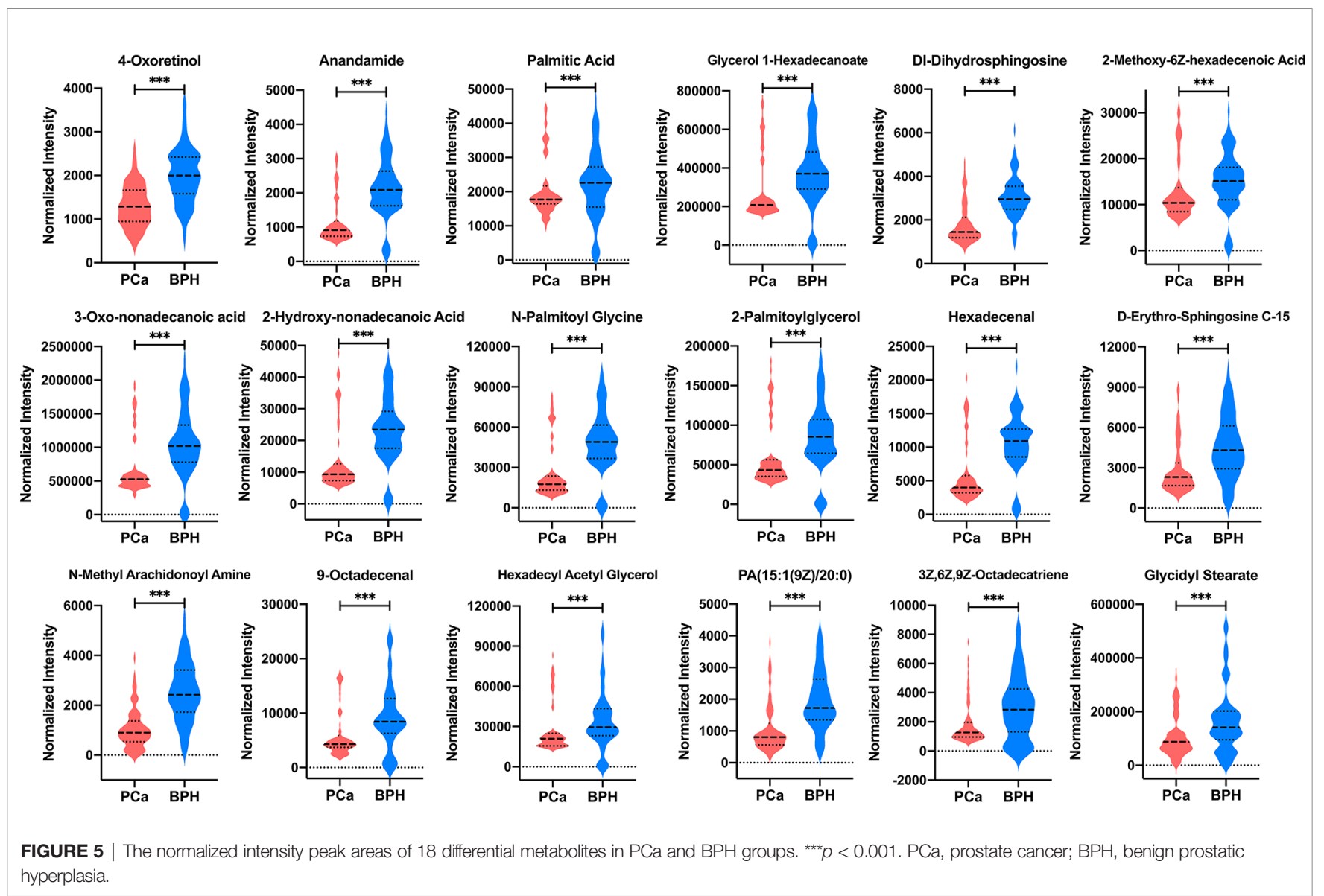




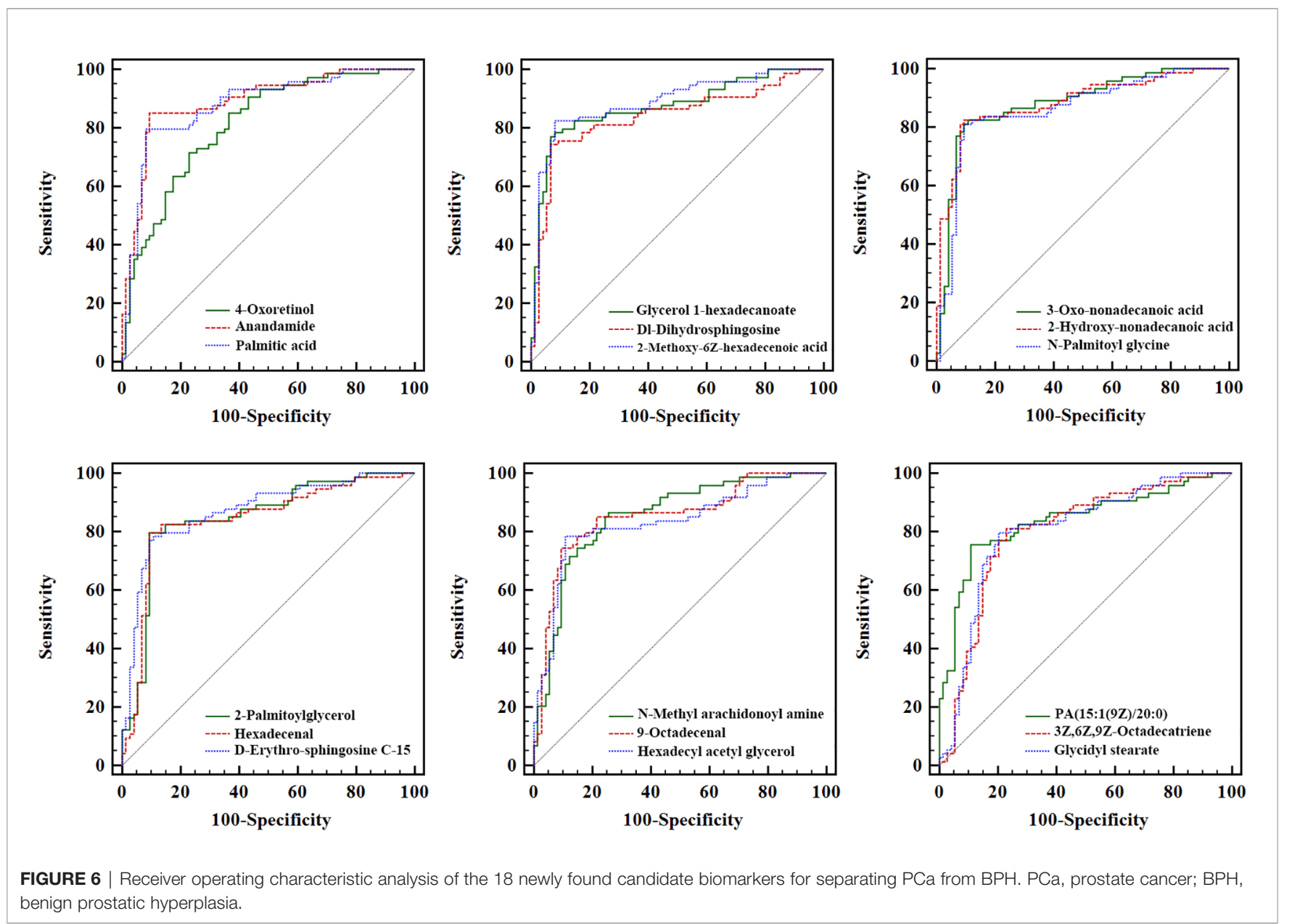

indicated that the 18 metabolites effectively discriminated $\mathrm{PCa}$ from BPH (AUC > 0.80).

\section{Correlations Between Differential Metabolites and Serum Fasting Lipid Profiles}

Levels of TC, HDL-C, LDL-C, and Apo-A1 were statistically significantly different among $\mathrm{HC}, \mathrm{BPH}$, and $\mathrm{PCa}$ groups (all $p<$ 0.05) (Table 4). Further analysis indicated that the levels of commonly used fasting lipid profiles including TC, TG, HDL-C,
LDL-C, and Apo-A1 (except Apo-B) were significantly different in $\mathrm{PCa}$ group compared with the levels in $\mathrm{HC}$ subjects. These findings indicated a potential association between lipid metabolism and occurrence and progression of PCa. Notably, differences in the levels of these clinical lipid parameters were not statistically significant between HC and $\mathrm{BPH}$ groups, implying that these profiles are not involved in prostatitis. Moreover, significantly higher serum concentrations of HDL-C and Apo-A1 were observed in $\mathrm{PCa}$ patients compared with the levels in $\mathrm{BPH}$ patients.

TABLE 4 | Serum lipids and apolipoproteins profile for participants.

\begin{tabular}{|c|c|c|c|c|}
\hline Fasting lipid profiles & $H C(n=72)$ & $\mathrm{BPH}(n=74)$ & $\mathrm{PCa}(\mathrm{n}=74)$ & $\mathbf{F}, p$ \\
\hline $\mathrm{TC}(\mathrm{mmol} / \mathrm{L})$ & $4.97 \pm 0.91$ & $4.95 \pm 0.99$ & $5.38 \pm 1.48^{\star \star \star}$ & $9.06,<0.001$ \\
\hline $\mathrm{TG}(\mathrm{mmol} / \mathrm{L})$ & $1.77 \pm 1.40$ & $1.87 \pm 1.49$ & $1.53 \pm 1.02^{\star}$ & $2.37,0.941$ \\
\hline HDL-C (mmol/L) & $1.42 \pm 0.32$ & $1.33 \pm 0.26$ & $1.49 \pm 0.32^{\star}$ & $5.49,0.004$ \\
\hline LDL-C (mmol/L) & $3.04 \pm 0.79$ & $2.99 \pm 0.72$ & $3.29 \pm 1.27^{\star \star}$ & $4.60,0.010$ \\
\hline Apo-A1 (g/L) & $1.48 \pm 0.23$ & $1.42 \pm 0.22$ & $1.56 \pm 0.25^{\star \star}$ & $8.54,<0.001$ \\
\hline Apo-B (g/L) & $1.00 \pm 0.24$ & $0.98 \pm 0.22$ & $1.04 \pm 0.31$ & $1.30,0.272$ \\
\hline
\end{tabular}

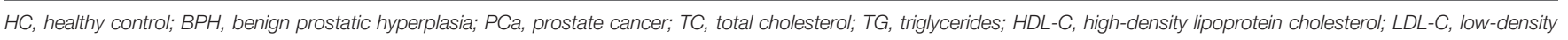
lipoprotein cholesterol.

Compared with healthy controls, ${ }^{* * *} p<0.001$, ${ }^{* *} \mathrm{p}<0.01$, ${ }^{*} \mathrm{p}<0.05$.

Compared with BPH subjects, ${ }^{\# \# \#} \mathrm{p}<0.001,{ }^{\# \#} \mathrm{p}<0.01$. 
These findings indicated significant changes in lipid metabolism in cancer patients.

Furthermore, correlation analysis was performed to explore the relationship between the 18 lipid or lipid-like metabolites and the commonly used fasting lipid profiles. All the 18 lipid-related metabolites were significantly negatively correlated with TC, LDL-C, and Apo-B (all $p<0.05$ ) (Table 5). Out of the 18 metabolites, four (4-oxoretinol, anandamide, palmitic acid, and glycerol 1-hexadecanoate) were negatively associated with HDL-C (all $p<0.05$ ), whereas the other metabolites, including DL-dihydrosphingosine, 2-methoxy-6Z-hexadecenoic acid, and $N$-palmitoyl glycine, had no correlation with it. Moreover, Apo-A1 exhibited a negative correlation with lipid profiles such as 4-oxoretinol, palmitic acid, and glycerol 1-hexadecanoate but was not correlated with anandamide, DL-dihydrosphingosine, 9octadecenal, 3Z,6Z,9Z-octadecatriene, and glycidyl stearate. Notably, TG was not correlated with all the 18 candidate biomarkers (all $p>0.05$ ), implying that the newly identified lipid biomarkers are not involved in the TG metabolism pathway.

\section{DISCUSSION}

Patients classified in the PSA level arrangement gray zone with moderate Gleason sum scores are characterized by low or moderate risk. Therefore, their therapeutics must be tailored ideally according to the tumor characteristics (14). In this condition, "active surveillance," which is increasingly recognized as initial treatment approach for men with low-risk PCa, is introduced $(26,27)$. However, repeated PSA assessments coupled with re-biopsies are required, which are associated with additional costs and risks. Currently, several biomarkers are available for early diagnosis of PCa (28); however, they are not effective for discriminating patients with $\mathrm{PCa}$ and $\mathrm{BPH}$ in the
PSA gray zone of $4-10 \mathrm{ng} / \mathrm{ml}$. Therefore, the present study sought to determine serum metabolic profiles between $\mathrm{PCa}$ and $\mathrm{BPH}$ patients with PSA levels at $4-10 \mathrm{ng} / \mathrm{ml}$, for use as potential non-invasive candidate biomarkers for accurate PCa diagnosis.

Dependence of androgens is an important hallmark of PCa. Androgens are implicated in regulation of lipid metabolism through the sterol regulatory element-binding proteins (SREBPs) associated pathway (29). Dysregulation of lipid metabolism affects several cellular processes such as proliferation, differentiation, and motility, which are highly involved in carcinogenesis pathways (such as transformation, progression, and metastasis) $(30,31)$. In the current study, dysregulation of lipid metabolism within the PSA gray zone of 4-10 ng/ml in PCa patients compared with BPH patients was firstly explored by LC-MS/MS analysis. Significant differences in glycerophospholipids, glycerolipid, and arachidonic acid metabolisms were observed between the two groups. Consistent with our findings, previous studies have reported the same dysregulation of glycerophospholipids, glycerolipid, and arachidonic acid metabolisms in various cancers (30), including lung cancer (32), breast cancer (33), colorectal cancer (34), ovarian cancer (35), pancreatic cancer (36), kidney cancer (37), and gastric cancer (13). A total of 18 lipid-related metabolites (including 4-oxoretinol, anandamide, palmitic acid, and glycidyl stearate) was identified using the selection criteria of variable VIP $>2$, FC $>1.5$ or $<2 / 3$, and $p<0.05$. These metabolites were downregulated in PCa patients compared with the BPH control subjects. The significant decrease in lipid profiles in PCa patients can be attributed to increased lipid utilization by neoplastic cells for membrane biogenesis (a characteristic of carcinogenesis), and increased requirement of phospholipids during fast cell proliferation $(13,38)$. Notably, dysregulated lipid metabolism can be caused by physiological

TABLE 5 | Correlation analysis of differential metabolites and commonly used fasting lipid profiles.

\begin{tabular}{|c|c|c|c|c|c|c|}
\hline Biomarker & $\mathrm{TC}(r, p)$ & $\mathrm{TG}(r, p)$ & HDL-C $(r, p)$ & LDL-C $(r, p)$ & Apo-A1 $(r, p)$ & Apo-B $(r, p)$ \\
\hline 4-Oxoretinol & $-0.578,<0.001$ & $0.031,0.794$ & $-0.242,0.038$ & $-0.502,<0.001$ & $-0.241,0.038$ & $-0.313,0.007$ \\
\hline Anandamide & $-0.542,<0.001$ & $0.033,0.782$ & $-0.245,0.035$ & $-0.571,<0.001$ & $-0.214,0.067$ & $-0.376,0.001$ \\
\hline Palmitic acid & $-0.561,<0.001$ & $-0.094,0.426$ & $-0.255,0.028$ & $-0.544,<0.001$ & $-0.294,0.011$ & $-0.481,<0.001$ \\
\hline Glycerol 1-hexadecanoate & $-0.591,<0.001$ & $-0.027,0.822$ & $-0.295,0.011$ & $-0.440,<0.001$ & $-0.354,0.002$ & $-0.285,0.014$ \\
\hline DL-Dihydrosphingosine & $-0.283,0.015$ & $-0.057,0.631$ & $-0.076,0.519$ & $-0.412,<0.001$ & $-0.083,0.483$ & $-0.429,<0.001$ \\
\hline 2-Methoxy-6Z-hexadecenoic acid & $-0.587,<0.001$ & $-0.079,0.504$ & $-0.215,0.066$ & $-0.527,<0.001$ & $-0.295,0.011$ & $-0.424,<0.001$ \\
\hline 3-Oxo-nonadecanoic acid & $-0.582,<0.001$ & $-0.081,0.491$ & $-0.246,0.035$ & $-0.463,<0.001$ & $-0.329,0.004$ & $-0.324,0.005$ \\
\hline 2-Hydroxy-nonadecanoic acid & $-0.566,<0.001$ & $-0.092,0.436$ & $-0.269,0.020$ & $-0.435,<0.001$ & $-0.359,0.002$ & $-0.280,0.016$ \\
\hline N-Palmitoyl glycine & $-0.524,<0.001$ & $-0.076,0.521$ & $-0.223,0.056$ & $-0.459,<0.001$ & $-0.240,0.039$ & $-0.314,0.007$ \\
\hline 2-Palmitoylglycerol & $-0.483,<0.001$ & $-0.174,0.139$ & $-0.191,0.103$ & $-0.459,<0.001$ & $-0.277,0.017$ & $-0.360,0.002$ \\
\hline Hexadecenal & $-0.576,<0.001$ & $-0.124,0.291$ & $-0.272,0.019$ & $-0.461,<0.001$ & $-0.319,0.006$ & $-0.304,0.008$ \\
\hline D-Erythro-sphingosine C-15 & $-0.569,<0.001$ & $-0.122,0.299$ & $-0.225,0.054$ & $-0.445,<0.001$ & $-0.266,0.022$ & $-0.361,0.002$ \\
\hline N-Methyl arachidonoyl amine & $-0.552,<0.001$ & $-0.055,0.641$ & $-0.242,0.038$ & $-0.475,<0.001$ & $-0.281,0.015$ & $-0.279,0.016$ \\
\hline 9-Octadecenal & $-0.461,<0.001$ & $-0.150,0.203$ & $-0.063,0.596$ & $-0.489,<0.001$ & $-0.111,0.347$ & $-0.445,<0.001$ \\
\hline Hexadecyl acetyl glycerol & $-0.474,<0.001$ & $-0.038,0.746$ & $-0.242,0.038$ & $-0.426,<0.001$ & $-0.280,0.016$ & $-0.326,0.005$ \\
\hline PA (15:1(9Z)/20:0) & $-0.549,<0.001$ & $0.067,0.570$ & $-0.283,0.015$ & $-0.458,<0.001$ & $-0.231,0.047$ & $-0.305,0.008$ \\
\hline 3Z,6Z,9Z-Octadecatriene & $-0.416,<0.001$ & $-0.024,0.839$ & $-0.075,0.525$ & $-0.409,<0.001$ & $-0.069,0.561$ & $-0.360,0.002$ \\
\hline Glycidyl stearate & $-0.463,<0.001$ & $-0.152,0.196$ & $-0.066,0.575$ & $-0.484,<0.001$ & $-0.124,0.292$ & $-0.484,<0.001$ \\
\hline
\end{tabular}

TC, total cholesterol; TG, triglycerides; HDL-C, high-density lipoprotein cholesterol; LDL-C, low-density lipoprotein cholesterol. 
factors of PCa subjects, such as hormone generation and expression of oncogenes (39). Androgens induce transcription of enzymes to activate lipid metabolism in PCa by inducing SREBP1 transcription factor $(40,41)$. Additionally, lipid metabolism-related oncogenes such as P53, fatty acid transporting genes (FABP), lipogenic genes (FAS), and lipolytic genes (ATGL) are implicated in regulation of metabolic processes, including glycolysis and lipogenesis (42, 43). However, the metabolic profile differences in PCa with various gene polymorphisms were not explored in the current study owing to lack of sufficient patient information. Therefore, further studies should focus on the metabolic profiles of different gene expressions.

Lipid metabolism with elevated cholesterol synthesis and steroid genesis favor development of PCa disease compared with other solid tumors $(40,44)$. In the current study, significantly higher levels of TC, LDL-C, and HDL-C were observed in PCa patients compared with those in $\mathrm{HC}$ and $\mathrm{BPH}$ controls. All the 18 newly identified metabolites were significantly negatively correlated with TC, LDL-C, and Apo-B levels in PCa patients, whereas only a few metabolites were negatively correlated with HDL-C and Apo-A levels. Analysis showed no correlation between TG level and the 18 candidate biomarkers, indicating that the metabolites were not implicated in TG metabolism. We speculated that TG present in circulation may not exert significant effects on the pathogenesis and progression of $\mathrm{PCa}$, but additional research is warranted. Lipids are currently used as effective biomarkers for several diseases in various clinical applications (13). In the present study, all the 18 metabolites hold the promise as alternative diagnostic tools for discrimination of $\mathrm{PCa}$ from $\mathrm{BPH}$ at the gray zone of PSA $4-10 \mathrm{ng} / \mathrm{ml}$ (all AUC > 0.80).

Potential application of metabolomics in PCa has attracted great attention following an unbiased metabolomics analysis by Sreekumar et al. The researchers found that metabolites including sarcosine, uracil, kynurenine, glycerol-3-phosphate, leucine, and proline exhibit high levels during PCa progression from benign adjacent prostate $(n=16)$ to localized PCa $(n=12)$ to metastatic PCa $(n=14)(45)$. Sarcosine displayed the most significant differences, with elevated levels in $79 \%$ of metastatic tumor tissues and $42 \%$ of localized tumor tissues and absent in benign specimens. In addition, McDunn et al. analyzed metabolites in two cohorts with 331 PCa tissue samples and 178 benign tissue (matched to 178 of the $\mathrm{PCa}$ specimens) samples using GC-MS and LC-MS/MS methods (46). Almost all of the significantly altered metabolites reported by Sreekumar et al. were observed in the study by McDunn et al., including high levels of the progression-associated metabolites glycerol-3phosphate, kynurenine, proline, threonine, and uracil in $\mathrm{PCa}$ tissue compared with the levels in benign tissue. But under the findings of McDunn et al., the sarcosine level was only significantly raised in tissue samples with a Gleason score of 8 or higher. Metabolomics studies have further reported elevated alterations of sarcosine levels in urine/serum/plasma samples of PCa patients $(18,19)$. In summary, these findings suggest that sarcosine is a potential effective biomarker for $\mathrm{PCa}$ and can be used for differentiation of high-grade PCa from low-grade PCa. In addition to the in-depth study of sarcosine, dysregulation of lipid metabolism in PCa is also extensively explored as a promising approach for assessing disease progression and outcome. For example, Lin et al. studied the correlation between the plasma lipid profiles and clinical outcomes in 96 patients with castrate-refractory PCa. A three-lipid signature comprising ceramide d18:1/24:1, sphingomyelin d18:2/16:0, and phosphatidylcholine 16:0/16:0 was established. Eleven subjects from the validation cohort (63 patients) exhibited the three-lipid signature and had a significant shorter median overall survival (11.3 months) than did patients who did not exhibit the signature (21.4 months) (47). These findings show significant progress in use of lipid metabolites for PCa prognosis. Moreover, fatty acids are catabolized by fatty acid oxidation, which is an essential energy source for abnormal cell proliferation (19); thereby, dysregulation of fatty acid metabolism pathway is vital in the development of cancers. Furthermore, alterations in energetic metabolism are common in tumors. Increased serum glucose level is correlated with an increased risk of $\mathrm{PCa}$ recurrence after radical resection or radiation therapy (19). Despite the promising data and the numerous urinary and serum biomarkers under investigation for PCa diagnosis, many critical issues should be covered in the near future to implement their use in clinical practice.

In summary, the present study provides a novel insight into PCa diagnosis in the gray zone of $4 \sim 10 \mathrm{ng} / \mathrm{ml}$. These newly found biomarkers would manage PCa patients by guiding biopsy and improving on the false-positive rate of PSA (48). Furthermore, combining blood biomarkers with multiparametric magnetic resonance imaging (mpMRI) would be of particular value for PCa management in clinic (49). However, the study had some limitations. Firstly, the study cohort size was small and lacked an external validation cohort; therefore, the models may have overfitting bias. Secondly, onetime measurement of metabolites and processing delays in sample preparation may increase risk of measurement error, thus affecting associations of metabolites with PCa risk. Thirdly, a multi-omics analysis integrating data on genes, proteins, and metabolites should be performed to gain deeper insights into the potential mechanisms of metabolites in modulating occurrence and progression of PCa.

\section{CONCLUSION}

Analysis of serum samples through LC-MS/MS-based metabolomics successfully discriminated patients and controls with $\mathrm{BPH}$ at the gray zone of PSA $4 \sim 10 \mathrm{ng} / \mathrm{ml}$. Significantly enriched pathways including glycerophospholipid, glycerolipid, and arachidonic acid metabolisms were observed in $\mathrm{PCa}$ patients. Eighteen novel lipid or lipid-like metabolites were identified as potential biomarkers for distinguishing $\mathrm{PCa}$ patients from BPH cases in the PSA gray zone of $4-10 \mathrm{ng} / \mathrm{ml}$. These findings provide a basis for addressing the current medical challenge of poor specificity of serum PSA for diagnosis of PCa. 


\section{DATA AVAILABILITY STATEMENT}

The datasets during and/or analyzed during the current study are available from the corresponding author on reasonable request.

\section{ETHICS STATEMENT}

The study was approved by the Medical Ethics Committee of Mianyang Central Hospital (approval no. P2020040). All patients provided written informed consent to participate.

\section{AUTHOR CONTRIBUTIONS}

All authors contributed to the study conception and design and take responsibility for the integrity of the data and the accuracy of the data analysis. Data collection was performed by BX, LG, and YZ; and analysis was performed by XC and YC. Material

\section{REFERENCES}

1. Lima AR, Pinto J, Amaro F, Bastos ML, Carvalho M, Guedes de Pinho P. Advances and Perspectives in Prostate Cancer Biomarker Discovery in the Last 5 Years Through Tissue and Urine Metabolomics. Metabolites (2021) 11 (3):181. doi: 10.3390/metabo11030181

2. Tsoi TH, Chan CF, Chan WL, Chiu KF, Wong WT, Ng CF, et al. Urinary Polyamines: A Pilot Study on Their Roles as Prostate Cancer Detection Biomarkers. PloS One (2016) 11(9):e0162217. doi: 10.1371/journal.pone.0162217

3. Bruzzone C, Loizaga-Iriarte A, Sánchez-Mosquera P, Gil-Redondo R, Astobiza I, Diercks T, et al. 1h-NMR-Based Urine Metabolomics Reveals Signs of Enhanced Carbon and Nitrogen Recycling in Prostate Cancer. J Proteome Res (2020) 19(6):2419-28. doi: 10.1021/acs.jproteome.0c00091

4. Liu J, Tang JL, Gong DX, Kong CZ. Level Change of Prostate-Specific Antigen in Patients With Benign Prostatic Hyperplasia After Transurethral Prostatic Resection. J Integr Nephrol Androl (2017) 4(1):10-3. doi: 10.4103/2394-2916.201276

5. Rigau M, Olivan M, Garcia M, Sequeiros T, Montes M, Colás E, et al. The Present and Future of Prostate Cancer Urine Biomarkers. Int J Mol Sci (2013) 14(6):12620-49. doi: 10.3390/ijms140612620

6. Wagenlehner FME, Oostrum EV, Tenke P, Tandogdu Z, Çek M, Grabe M, et al. Infective Complications After Prostate Biopsy: Outcome of the Global Prevalence Study of Infections in Urology (GPIU) 2010 and 2011, A Prospective Multinational Multicentre Prostate Biopsy Study. Eur Urol (2013) 63(3):521-7. doi: 10.1016/j.eururo.2012.06.003

7. Chan ES, Lo KL, Ng CF, Hou SM, Yip SK. Randomized Controlled Trial of Antibiotic Prophylaxis Regimens for Transrectal Ultrasound-Guided Prostate Biopsy. Chin Med J (2012) 125(14):2432-5. doi: 10.3760/cma.j.issn.0366-6999.2012.14.006

8. Giskeødegård GF, Hansen AF, Bertilsson H, Gonzalez SV, Kristiansen KA, Bruheim P, et al. Metabolic Markers in Blood Can Separate Prostate Cancer From Benign Prostatic Hyperplasia. Br J Cancer (2015) 113(12):1712-9. doi: 10.1038/bjc.2015.411

9. Cairns RA, Harris IS, Mak TW. Regulation of Cancer Cell Metabolism. Nat Rev Cancer (2011) 11(2):85-95. doi: 10.1038/nrc2981

10. Zhang AH, Sun H, Wang XJ. Serum Metabolomics as A Novel Diagnostic Approach for Disease: A Systematic Review. Anal Bioanal Chem (2012) 404 (4):1239-45. doi: 10.1007/s00216-012-6117-1

11. Ni Y, Xie G, Jia W. Metabonomics of Human Colorectal Cancer: New Approaches for Early Diagnosis and Biomarker Discovery. J Proteome Res (2014) 13(9):3857-70. doi: 10.1021/pr500443c

12. Tao L, Zhou J, Yuan C, Zhang L, Li D, Si D, et al. Metabolomics Identifies Serum and Exosomes Metabolite Markers of Pancreatic Cancer. Metabolomics (2019) 15:86. doi: 10.1007/s11306-019-1550-1 preparation and the first draft of the manuscript was written by JF and LY; and all authors commented on previous versions of the manuscript. All authors contributed to the article and approved the submitted version.

\section{FUNDING}

This work was financially supported by the Sichuan Health and Health Committee Support Program (20PJ255), Incubation Project of Mianyang Central Hospital (2019FH01 and 2019YJ22), and CSCO-qilu cancer scientific research fund (NO.Y-QL202101-0125).

\section{SUPPLEMENTARY MATERIAL}

The Supplementary Material for this article can be found online at: https://www.frontiersin.org/articles/10.3389/fonc. 2021. 730638/full\#supplementary-material

13. Yu L, Lai Q, Feng Q, Li Y, Feng J, Xu B. Serum Metabolic Profiling Analysis of Chronic Gastritis and Gastric Cancer by Untargeted Metabolomics. Front Oncol (2021) 11:636917. doi: 10.3389/fonc.2021.636917

14. Kdadra M, Höckner S, Leung H, Kremer W, Schiffer E. Metabolomics Biomarkers of Prostate Cancer: A Systematic Review. Diagnostics (Basel) (2019) 9(1):21. doi: 10.3390/diagnostics9010021

15. Gómez-Cebrián N, Rojas-Benedicto A, Albors-Vaquer A, López-Guerrero JA, Pineda-Lucena A, Puchades-Carrasco L. Metabolomics Contributions to the Discovery of Prostate Cancer Biomarkers. Metabolites (2019) 9(3):48. doi: 10.3390/metabo9030048

16. Eidelman E, Twum-Ampofo J, Ansari J, Siddiqui MM. The Metabolic Phenotype of Prostate Cancer. Front Oncol (2017) 7:131. doi: 10.3389/ fonc.2017.00131

17. Lima AR, Araújo AM, Pinto J, Jerónimo C, Henrique R, Bastos ML, et al. GCMS-Based Endometabolome Analysis Differentiates Prostate Cancer From Normal Prostate Cells. Metabolites (2018) 8(1):23. doi: 10.3390/ metabo8010023

18. Trock BJ. Application of Metabolomics to Prostate Cancer. Urol Oncol (2011) 29(5):572-81. doi: 10.1016/j.urolonc.2011.08.002

19. Lima AR, Bastos Mde L, Carvalho M, Guedes de Pinho P. Biomarker Discovery in Human Prostate Cancer: An Update in Metabolomics Studies. Transl Oncol (2016) 9(4):357-70. doi: 10.1016/j.tranon.2016.05.004

20. Röhnisch HE, Kyrø C, Olsen A, Thysell E, Hallmans G, Moazzami AA. Identification of Metabolites Associated With Prostate Cancer Risk: A Nested Case Control Study With Long Follow-Up in the Northern Sweden Health and Disease Study. BMC Med (2020) 18(1):187. doi: 10.1186/s12916-020-01655-1

21. Huang Y, Niu M, Jing J, Zhang ZT, Zhao X, Chen SS, et al. Metabolomic Analysis Uncovers Energy Supply Disturbance as an Underlying Mechanism of the Development of Alcohol-Associated Liver Cirrhosis. Hepatol Commun (2021) 5(6):961-75. doi: 10.1002/hep4.1699

22. Chaleckis R, Meister I, Zhang P, Wheelock CE. Challenges, Progress and Promises of Metabolite Annotation for LC-MS-Based Metabolomics. Curr Opin Biotechnol (2019) 55:44-50. doi: 10.1016/j.copbio.2018.07.010

23. Viant MR, Kurland IJ, Jones MR, Dunn WB. How Close Are We to Complete Annotation of Metabolomes? Curr Opin Chem Biol (2017) 36:64-9. doi: 10.1016/j.cbpa.2017.01.001

24. Luo P, Yin P, Hua R, Tan Y, Li Z, Qiu G, et al. A Large-Scale, Multicenter Serum Metabolite Biomarkers Identification Study for the Early Detection of Hepatocellular Carcinoma. Hepatology (2018) 67(2):662-75. doi: 10.1002/ hep. 29561

25. Chen Y, Ma Z, Zhong J, Li L, Min L, Xu L, et al. Simultaneous Quantification of Serum Monounsaturated and Polyunsaturated Phosphatidylcholines as 
Potential Biomarkers for Diagnosing Non-Small Cell Lung Cancer. Sci Rep (2018) 8(1):7137. doi: 10.1038/s41598-018-25552-z

26. Briganti A, Fossati N, Catto JWF, Cornford P, Montorsi F, Mottet N, et al. Active Surveillance for Low-Risk Prostate Cancer: The European Association of Urology Position in 2018. Eur Urol (2018) 74(3):357-68. doi: 10.1016/ j.eururo.2018.06.008

27. Seaman AT, Taylor KL, Davis K, Nepple KG, Lynch JH, Oberle AD, et al. Why Men With A Low-Risk Prostate Cancer Select and Stay on Active Surveillance: A Qualitative Study. PloS One (2019) 14(11):e0225134. doi: 10.1371/ journal.pone.0225134

28. Kiebish MA, Cullen J, Mishra P, Ali A, Milliman E, Rodrigues LO, et al. MultiOmic Serum Biomarkers for Prognosis Of Disease Progression in Prostate Cancer. J Transl Med (2020) 18(1):10. doi: 10.1186/s12967-019-02185-y

29. Butler LM, Centenera MM, Swinnen JV. Androgen Control of Lipid Metabolism in Prostate Cancer: Novel Insights and Future Applications. Endocr Relat Cancer (2016) 23(5):R219-27. doi: 10.1530/ERC-15-0556

30. Perrotti F, Rosa C, Cicalini I, Sacchetta P, Del Boccio P, Genovesi D, et al. Advances in Lipidomics for Cancer Biomarkers Discovery. Int J Mol Sci (2016) 17(12):1992. doi: 10.3390/ijms17121992

31. Shevchenko A, Simons K. Lipidomics: Coming to Grips With Lipid Diversity. Nat Rev Mol Cell Biol (2010) 11(8):593-8. doi: 10.1038/nrm2934

32. Hall Z, Ament Z, Wilson CH, Burkhart DL, Ashmore T, Koulman A, et al. Expression Drives Aberrant Lipid Metabolism in Lung Cancer. Cancer Res (2016) 76(16):4608-18. doi: 10.1158/0008-5472.CAN-15-3403

33. Kang HS, Lee SC, Park YS, Jeon YE, Lee JH, Jung SY, et al. Protein and Lipid MALDI Profiles Classify Breast Cancers According to the Intrinsic Subtype. BMC Cancer (2011) 11:465-74. doi: 10.1186/1471-2407-11-465

34. Martín-Blázquez A, Díaz C, González-Flores E, et al. Untargeted LC-HRMSBased Metabolomics to Identify Novel Biomarkers of Metastatic Colorectal Cancer. Sci Rep (2019) 9(1):20198. doi: 10.1038/s41598-019-55952-8

35. Buas MF, Gu H, Djukovic D, Zhu J, Drescher CW, Urban N, et al. Identification of Novel Candidate Plasma Metabolite Biomarkers for Distinguishing Serous Ovarian Carcinoma and Benign Serous Ovarian Tumors. Gynecol Oncol (2016) 140(1):138-44. doi: 10.1016/j.ygyno.2015.10.021

36. Shu X, Zheng W, Yu D, Li HL, Lan Q, Yang G, et al. Prospective Metabolomics Study Identifies Potential Novel Blood Metabolites Associated With Pancreatic Cancer Risk. Int J Cancer (2018) 143(9):2161-7. doi: 10.1002/ ijc.31574

37. Saito K, Arai E, Maekawa K, Ishikawa M, Fujimoto H, Taguchi R, et al. Lipidomic Signatures and Associated Transcriptomic Profiles of Clear Cell Renal Cell Carcinoma. Sci Rep (2016) 6:28932. doi: 10.1038/srep28932

38. Lee JW, Kim EY, Yoo HM, Park CH, Song KY. Changes of Lipid Profiles After Radical Gastrectomy in Patients With Gastric Cancer. Lipids Health Dis (2015) 14:21. doi: 10.1186/s12944-015-0018-1

39. Xu H, Chen J, He J, Ji J, Cao Z, Chen X, et al. Serum Metabolic Profiling Identifies a Biomarker Panel for Improvement of Prostate Cancer Diagnosis. Front Oncol (2021) 11:666320. doi: 10.3389/fonc.2021.666320

40. Chen M, Zhang J, Sampieri K, Clohessy JG, Mendez L, Gonzalez-Billalabeitia E, et al. An Aberrant SREBP-Dependent Lipogenic Program Promotes Metastatic Prostate Cancer. Nat Genet (2018) 50(2):206-18. doi: 10.1038/ s41588-017-0027-2
41. Tennakoon JB, Shi Y, Han JJ, Tsouko E, White MA, Burns AR, et al. Androgens Regulate Prostate Cancer Cell Growth Via an AMPK-PGC1alpha-Mediated Metabolic Switch. Oncogene (2014) 33(45):5251-61. doi: 10.1038/onc.2013.463

42. Napoli M, Flores ER. The P53 Family Orchestrates the Regulation of Metabolism: Physiological Regulation and Implications for Cancer Therapy. Br J Cancer (2017) 116(2):149-55. doi: 10.1038/bjc.2016.384

43. Carbonetti G, Wilpshaar T, Kroonen J, Studholme K, Converso C, d'Oelsnitz $\mathrm{S}$, et al. FABP5 Coordinates Lipid Signaling That Promotes Prostate Cancer Metastasis. Sci Rep (2019) 9(1):18944. doi: 10.1038/s41598-019-55418-x

44. Mitsuzuka K, Arai Y. Metabolic Changes in Patients With Prostate Cancer During Androgen Deprivation Therapy. Int J Urol (2018) 25(1):45-53. doi: 10.1111/iju.13473

45. Sreekumar A, Poisson LM, Rajendiran TM, Khan AP, Cao Q, Yu J, et al. Metabolomic Profiles Delineate Potential Role for Sarcosine in Prostate Cancer Progression. Nature (2009) 457(7231):910-4. doi: 10.1038/nature07762

46. McDunn JE, Li Z, Adam KP, Neri BP, Wolfert RL, Milburn MV, et al. Metabolomic Signatures of Aggressive Prostate Cancer. Prostate (2013) 73 (14):1547-60. doi: 10.1002/pros.22704

47. Lin HM, Mahon KL, Weir JM, Mundra PA, Spielman C, Briscoe K, et al. A Distinct Plasma Lipid Signature Associated With Poor Prognosis in Castration-Resistant Prostate Cancer: Prognostic Lipid Signature in Metastatic Prostate Cancer. Int J Cancer (2017) 141(10):2112-20. doi: $10.1002 / \mathrm{ijc} .30903$

48. Carrion DM, Gómez Rivas J, Álvarez-Maestro M, Martínez-Piñeiro L. Biomarkers in Prostate Cancer Management. Is There Something New? Arch Esp Urol (2019) 72(2):105-15.

49. Salciccia S, Capriotti AL, Laganà A, Fais S, Logozzi M, De Berardinis E, et al. Biomarkers in Prostate Cancer Diagnosis: From Current Knowledge to the Role of Metabolomics and Exosomes. Int J Mol Sci (2021) 22(9):4367. doi: 10.3390/ijms22094367

Conflict of Interest: Author XC was employed by SCIEX Analytical Instrument Trading Co.

The remaining authors declare that the research was conducted in the absence of any commercial or financial relationships that could be construed as a potential conflict of interest.

Publisher's Note: All claims expressed in this article are solely those of the authors and do not necessarily represent those of their affiliated organizations, or those of the publisher, the editors and the reviewers. Any product that may be evaluated in this article, or claim that may be made by its manufacturer, is not guaranteed or endorsed by the publisher.

Copyright $\odot 2021 \mathrm{Xu}$, Chen, Chen, Gan, Zhang, Feng and Yu. This is an open-access article distributed under the terms of the Creative Commons Attribution License (CC BY). The use, distribution or reproduction in other forums is permitted, provided the original author(s) and the copyright owner(s) are credited and that the original publication in this journal is cited, in accordance with accepted academic practice. No use, distribution or reproduction is permitted which does not comply with these terms. 